\title{
Factores asociados con la sintomatología depresiva en adolescentes michoacanos. El papel de la migración familiar y los recursos individuales, familiares y sociales
}

\author{
María Elena Rivera-Heredia, ' Lizeth Guadalupe Martínez-Servín, ${ }^{1}$ Nydia Obregón-Velasco'
}

Artículo original

\begin{abstract}
SUMMARY
Introduction

In order to understand what is happening with adolescents' emotional lives, this research analyzed factors associated with depressive symptomatology taking into account their personal, social and family resources and the experience of migration on their family.

\section{Material and methods}

The sample included 360 adolescents $150.6 \%$ women and $49.4 \%$ men; mean age $=13.5, \mathrm{SD}=.68)$ from ten different regions of $\mathrm{Mi}$ choacan, Mexico. The instruments were CES-D-R $(\alpha=.93)$, Affective Resources Scale $(\alpha=.82)$, Social Resources Scale $(\alpha=.77)$, and Family Resources Scale $(\alpha=.81)$. The tests were performed in the classroom, with the agreement of the participants.
\end{abstract}

\section{Results}

A linear regression analysis was used. The main result was that $42 \%$ of variance of depressive symptomatology is explained by risk and protection factors. The risk factors were being a woman, difficulties in sadness management and in anger management, and inability to ask for help to the social support networks and migration of the mother. The protective factors were self-control and expression in the family.

\section{Conclusions}

The affective, social and family resources should be strengthened to promote wellbeing in adolescents.

Key words: Adolescents, CESD-R, depressive symptomatology, family, migration, psychological resources.

\section{RESUMEN}

\section{Introducción}

Con la intención de comprender qué está pasando con la vida emocional de los adolescentes, esta investigación pretende identificar los factores asociados con la sintomatología depresiva tomando en cuenta los recursos individuales, familiares y sociales, así como la experiencia de migración en la familia.

\section{Material y métodos}

La muestra fue seleccionada por cuota con un número equivalente de participantes de cada una de las diez regiones socioeconómicas de Michoacán. En total participaron 360 estudiantes del segundo grado de secundaria $(50.6 \%$ mujeres y $49.4 \%$ hombres), con una edad promedio de 13.5 años. Se utilizaron los siguientes instrumentos: CES-D-R $(\alpha=.93)$, Escala de Recursos Afectivos ( $\alpha=.82)$, Escala de Recursos Sociales $(\alpha=.77)$; y para medir los recursos familiares se utilizó la Escala de Evaluación de Interrelaciones Familiares [ERI] con una consistencia interna de $\alpha=.81$. Se realizó un análisis de regresión múltiple lineal.

\section{Resultados}

El resultado central fue que el $42 \%$ de la varianza de la sintomatología depresiva puede explicarse por los siguientes factores de riesgo y de protección. Entre los factores de riesgo se encuentran: ser mujer, las dificultades para manejar la tristeza y el enojo, así como la incapacidad para buscar ayuda con la red de apoyo social y la migración de la madre. Como factores de protección están: el autocontrol y la expresión en la familia.

\section{Conclusiones}

Por lo tanto, los recursos individuales, familiares y sociales deben fortalecerse para promover un mayor estado de bienestar psicosocial en los adolescentes.

Palabras clave: Adolescentes, CESD-R, familia, migración, recursos psicológicos, sintomatología depresiva.

Facultad de Psicología. Universidad Michoacana de San Nicolás de Hidalgo (UMNSH), Morelia, Mich. México.

Correspondencia: Dra. María Elena Rivera Heredia. Facultad de Psicología, UMNSH. Gral. Francisco Villa 450, Edificio A, col. Dr. Miguel Silva. 58120, Morelia, Michoacán, México. Tel: 01 (443) 317 - 8951 Ext. 126. Fax: 01 (443) 312 - 9913. E-mail: maelenarivera@hotmail.com; maelenarivera@gmail.com Recibido: 8 de marzo de 2012. Aceptado: 23 de noviembre de 2012. 


\section{INTRODUCCIÓN}

Según la Organización Mundial de la Salud ${ }^{1}$ aproximadamente 121 millones de personas en todo el mundo presentan depresión, la cual es un trastorno del estado de ánimo crónico o recurrente que afecta la habilidad para enfrentarse a la vida cotidiana y es una de las principales causas de discapacidad laboral. Los principales síntomas de la depresión son tristeza, pérdida de placer, sentimientos de culpa y baja autovaloración, cambios en el sueño y el apetito, disminución en la energía y en la concentración; se asocia con suicidios, homicidios, comportamientos agresivos y consumo de drogas. ${ }^{2}$

Aunque se reconoce la relevancia de la depresión como un problema de salud mental en el mundo y en México, es necesario intensificar los esfuerzos para que la población pueda ser atendida oportunamente. ${ }^{3}$ También es necesario trabajar en el diseño de políticas públicas que incrementen la disponibilidad y acceso de los servicios de salud mental dirigidos a los adolescentes. ${ }^{4}$

Para la detección temprana y oportuna de factores de riesgo de la depresión existen instrumentos de tamizaje que evalúan la sintomatología depresiva. Uno de los más reconocidos internacionalmente es la Escala de Depresión del Centro de Estudios Epidemiológicos (CES-D) ${ }^{5}$ así como la versión revisada de la misma (CESD-R). ${ }^{6,7}$

Aún cuando la depresión está presente en diferentes etapas del ciclo vital, ${ }^{8-10}$ uno de los periodos de mayor riesgo para su aparición es la adolescencia, dada la vulnerabilidad de esta etapa, ${ }^{11}$ además del riesgo de cronicidad de dicha enfermedad cuando se inicia a edades tempranas, pues más del 3\% de los adolescentes sufre condiciones depresivas tan graves que pueden ocasionar en ellos diversas disfunciones sociales. ${ }^{12}$

La sintomatología depresiva en adolescentes tiene características singulares como síntomas más agudos de desamparo, falta de placer, desesperación y cambios más grandes de peso que en los adultos; los episodios de depresión observados combinan periodos de funcionamiento inadecuado con fases de un buen funcionamiento, siendo más probable el abuso de sustancias, embarazos no deseados y conductas violentas, especialmente contra ellos mismos, por ejemplo con intentos de suicidio. ${ }^{13}$ Durante la adolescencia se ha encontrado asociación entre la sintomatología depresiva con la deserción escolar, la emocionalidad restrictiva, ${ }^{14}$ así como con diversos problemas de salud mental. . $^{15,16}$

En estudios realizados en México se reporta mayor sintomatología depresiva cuando hay un ambiente familiar conflictivo, con una comunicación hostil y de rechazo, así como con poca cohesión familiar. ${ }^{17}$ Por el contrario, aspectos que promueven un ajuste emocional positivo en los adolescentes y por lo tanto minimizan el riesgo de que se presente un trastorno depresivo son la presencia de una comunicación abierta y fluida entre padres e hijos, la expresión de emociones positivas, la unión y apoyo existente dentro de la familia o una organización clara de los padres hacia los hijos con respecto a las reglas de disciplina. ${ }^{18-21}$

En uno de los pocos estudios realizados en México que abordan los recursos psicológicos y la sintomatología depresiva se encontró que los jóvenes con intento suicida presentan menor desarrollo de sus recursos afectivos, cognitivos, sociales (dificultad para solicitar apoyo) y familiares (menor unión y apoyo familiar, menor expresión en la familia y mayor percepción de dificultades familiares), que los adolescentes $\sin$ intento suicida. ${ }^{22}$

Los recursos psicológicos son definidos como objetos, condiciones, características personales y elementos no tangibles que tienen por sí mismos un valor para la supervivencia, ya sea de manera directa o indirecta y sirven como medio para alcanzar nuevos recursos. Los recursos pueden ser conservados, obtenidos, protegidos, promovidos e intercambiados. ${ }^{23}$

La teoría de la conservación de los recursos ${ }^{24,25}$ explica el estrés a partir de cómo las personas obtienen, conservan, desarrollan y protegen sus recursos, tratando de adaptarse y encajar en los contextos en los que se encuentran, de manera que utilizan los recursos para regularse a sí mismos y a las relaciones sociales que establecen. Los recursos existen en "caravanas", es decir, que no se desarrollan aisladamente sino en grupo y bajo una sensibilidad especial al contexto, por lo que para comprender qué ocurre con los adolescentes cuando se enfrentan a situaciones estresantes, qué pasa con sus recursos psicológicos y qué ocurre con la presencia o no de sintomatología depresiva, es necesario comprender la conducta individual dentro del contexto, para lo que se requiere una perspectiva ecológica y multifactorial.

En síntesis, un adolescente que presente problemas de salud mental debe ser detectado de manera temprana pues requiere apoyo oportuno que le permita transitar por esta etapa sin mayores conflictos. En el momento actual ya se cuenta con resultados de diversas investigaciones sobre sintomatología depresiva en adolescentes mexicanos, sin embargo todavía no existen suficientes estudios que destaquen el papel de los recursos psicológicos, tanto individuales como familiares, o la relación de la sintomatología depresiva con diversos sucesos estresantes como sería el caso de la migración familiar; ${ }^{26,27}$ también son escasas las investigaciones que reporten el uso de la nueva versión revisada del CESD-R. ${ }^{28}$ En estos elementos está centrada la relevancia del presente trabajo.

El objetivo de esta investigación fue identificar los factores asociados con la sintomatología depresiva de los adolescentes michoacanos a partir de sus recursos psicológicos individuales (autocontrol, recuperación del equilibrio, manejo de la tristeza y manejo del enojo), sociales (red de apoyo social y capacidad para pedir apoyo a la red) y familiares (unión y apoyo, expresión y dificultades familiares), considerando la migración familiar. 


\section{MATERIAL Y MÉTODOS}

\section{Participantes}

En total participaron 360 estudiantes con una media de edad de 13.6 años ( $\mathrm{DE}=.67), 49.4 \%$ eran hombres y $50.6 \%$, mujeres; tenían una edad mínima de 12 años y máxima de 16. Los participantes se encontraban estudiando el segundo grado en una secundaria pública proveniente de cada una de las diez zonas socioeconómicas del Estado de Michoacán, cuyas localidades y número de participantes fueron: Región Lerma Chapala con Zamora (50); región Bajío con Zacapu (42); Cuitzeo con la ciudad de Morelia (48); Oriente con ciudad Hidalgo (34); Tepalcatepec con Apatzingán (34); Purépecha con Uruapan (20); PátzcuaroZirahuén, con Pátzcuaro (27); Tierra caliente con Huetamo (50); Sierra Costa con Lázaro Cárdenas (34) e Infiernillo con Ario de Rosales (21). La muestra fue no probabilística. El $34.4 \%$ de ellos manifestaron tener padre migrante, el $13.1 \%$ madre migrante y el $17.2 \%$ tenían por lo menos un hermano(a) migrante.

\section{Instrumentos}

Se retomaron dos escalas de recursos psicológicos desarrolladas y validadas por Rivera-Heredia y Andrade, ${ }^{22}$ la Escala de Recursos Afectivos $(\alpha=.82)$, que se consideró para evaluar los recursos individuales, y la de Recursos Sociales $(\alpha=.77)$. Para evaluar los recursos familiares se utilizó la Escala de Evaluación de las Relaciones Intrafamiliares (ERI) en su versión corta de doce reactivos $(\alpha=.91)$. La sintomatología depresiva se evaluó con la versión revisada de la Escala de Depresión del Centro de Estudios Epidemiológicos (CESD-R) con 35 reactivos, la cual fue desarrollada por Radloff, ${ }^{5}$ validada en México por Mariño, Medina-Mora, Chaparro y GonzálezForteza, ${ }^{29}$ y cuya versión revisada CESD-R ha demostrado ser válida y confiable en adolescentes estudiantes ${ }^{6}$ y personas de la tercera edad.?

Para mantener niveles adecuados de consistencia interna fue necesario eliminar algunos reactivos, como el 24 en la subescala de disforia, el 10 en la de anhedonia y el 22 en la de culpa excesiva. No se incluyeron en esta investigación aquellas subescalas que tenían menos de dos reactivos, como sería el caso de fatiga, apetito, retardo psicomotor y pensamiento. La subescala de ideación suicida se conformó con los reactivos 14 y 15 del CESD-R junto con tres reactivos adicionales que se han retomado en otras investigaciones sobre el tema. ${ }^{28,30} \mathrm{La}$ escala de CESD-R total se integró con 35 reactivos.

\section{Procedimiento}

Se solicitó permiso para ingresar a las dependencias educativas a través de la Secretaría de Educación en el Estado. Una vez que se tuvo su aprobación se contactó a los directivos de diez secundarias públicas. Los instrumentos fueron aplicados a los estudiantes dentro del salón de clase por psicólogas previamente entrenadas para ello. Se invitó a los estudiantes a participar de manera voluntaria, lo cual fue aceptado por ellos. Se enfatizó la confidencialidad del manejo de los datos proporcionados por los estudiantes. Las aplicadoras se desplazaron desde la ciudad de Morelia a las localidades que corresponden a cada una de las regiones participantes. La administración de los instrumentos transcurrió de manera tranquila y sin incidentes durante los meses de abril a junio de 2010.

Cuadro 1. Confiabilidad y ejemplos de reactivos de las subescalas del CESD-R y de los recursos individuales, sociales y familiares

\begin{tabular}{llc}
\hline & & Alfa de \\
Subescalas y ejemplos de reactivos & & Cronbach \\
\hline Sintomatología depresiva (total de reactivos del CESD-R) & .91 \\
1. Estado de ánimo deprimido (disforia) & No podía quitarme la tristeza & .91 \\
2. Disminución del placer (anhedonia) & Nada me hacía feliz & .84 \\
3. Culpa excesiva o inapropiada & Pensé que mi vida ha sido un fracaso & .65 \\
4. Aislamiento social (Amigos/ocupación/familia) & Hablé menos de lo usual & .77 \\
5. Ideación suicida & Sentía deseos de estar muertola) & .69 \\
Recursos individuales (total de reactivos de Recursos afectivos) & .91 \\
7. Autocontrol & Puedo enfrentar situaciones difíciles permaneciendo en calma \\
8. Recuperación del equilibrio & Darme cuenta de cómo me siento me ayuda a recuperarme de los \\
9. Dificultades para el manejo de la tristeza & problemas & .74 \\
10. Dificultades para el manejo del enojo & Cuando estoy muy triste siento que mis problemas no tienen solución & .73 \\
Recursos sociales (total de reactivos de Recursos afectivos) & Pierdo el control cuando me enojo & .48 \\
11. Red de apoyo & Por lo menos tengo a una persona a quien contarle lo que me pasa \\
12. Dificultades para solicitar apoyo & Para mí es muy difícil pedir ayuda a los demás & .71 \\
Recursos familiares (total de reactivos de Recursos familiares) & .68 \\
13. Unión y apoyo & Los miembros de la familia acostumbran hacer cosas juntos \\
14. Expresión & Mis padres me animan a expresar abiertamente mis puntos de vista \\
15. Dificultades & En mi familia, nadie se preocupa por los sentimientos de los demás & .84 \\
\hline
\end{tabular}


Cuadro 2. Explicación de la sintomatología depresiva mediante los recursos individuales, familiares y sociales, así como de la migración familiar utilizando el análisis de regresión múltiple

\begin{tabular}{|c|c|c|c|c|c|c|c|c|c|c|}
\hline \multirow[b]{2}{*}{ Modelo } & \multirow[b]{2}{*}{$\mathrm{R}$} & \multirow[b]{2}{*}{$\begin{array}{c}\mathrm{R} \\
\text { cuadrado }\end{array}$} & \multirow[b]{2}{*}{$\begin{array}{l}\text { R cuadrado } \\
\text { corregida }\end{array}$} & \multirow[b]{2}{*}{$\begin{array}{l}\text { Error } \\
\text { típico }\end{array}$} & \multicolumn{5}{|c|}{ Estadísticos de cambio } & \multirow[b]{2}{*}{$\begin{array}{l}\text { Durbin- } \\
\text { Watson }\end{array}$} \\
\hline & & & & & $\begin{array}{l}\text { Cambio en } \\
\text { R cuadrado }\end{array}$ & $\begin{array}{l}\text { Cambio } \\
\text { en } \mathrm{F}\end{array}$ & $\begin{array}{l}\text { Grados } \\
\text { de libertad }\end{array}$ & Beta & $\begin{array}{c}\text { Sig. Cambio } \\
\text { en } \mathrm{F}\end{array}$ & \\
\hline 1. & $.55 a$ & 31 & .30 & 19.98 & 31 & 150.52 & 1343 & .347 & .000 & \\
\hline 2. & $.61 b$ & .37 & .37 & 19.01 & .07 & 36.53 & 1342 & -.224 & .000 & \\
\hline 3. & $.62 c$ & .39 & .39 & 18.70 & .02 & 12.63 & 1341 & -.146 & .000 & \\
\hline 4. & $.64 d$ & .41 & .41 & 18.44 & .02 & 10.47 & 1340 & .151 & .001 & \\
\hline 5. & $.64 \mathrm{e}$ & .42 & .41 & 18.33 & .01 & 5.38 & 1339 & .122 & .021 & \\
\hline 6. & $.65 f$ & .43 & .42 & 18.22 & .01 & 4.95 & 1338 & .103 & .027 & \\
\hline 7. & $.66 \mathrm{~g}$ & .44 & .43 & 18.11 & .01 & 5.18 & 1337 & .093 & .023 & 1.930 \\
\hline
\end{tabular}

a. Variables predictoras:(Constante), dificultades para el manejo de la tristeza.

b. Variables predictoras:(Constante), dificultades para el manejo de la tristeza, autocontrol.

c. Variables predictoras:(Constante), dificultades para el manejo de la tristeza, autocontrol, expresión.

d. Variables predictoras:(Constante), dificultades para el manejo de la tristeza, autocontrol, expresión, dificultades para el manejo del enojo.

e. Variables predictoras:(Constante), dificultades en el manejo de la tristeza, autocontrol, expresión, dificultades para el manejo del enojo, incapacidad para buscar apoyo.

f. Variables predictoras:(Constante), dificultades en el manejo de la tristeza, autocontrol, expresión, dificultades para el manejo del enojo, incapacidad para buscar apoyo, sexo.

g. Variables predictoras:(Constante), dificultades en el manejo de la tristeza, autocontrol, expresión, dificultades para el manejo del enojo, incapacidad para buscar apoyo, sexo, madre migrante.

h. Variable dependiente: Puntaje total del CESD-R con 35 reactivos.

\section{Análisis de datos}

Se realizaron análisis de regresión múltiple por el método de pasos sucesivos, así como análisis de correlación de Pearson y de comparación de medias. Para ello se utilizó el paquete estadístico SPSS versión 17.0. ${ }^{31}$

\section{RESULTADOS}

La consistencia interna de las escalas y subescalas de la CESD$\mathrm{R}$ y de las Escalas de Recursos Individuales, Sociales y Familiares, obtenidas a partir de la muestra que participó en este estudio se muestran en el cuadro 1, las cuales están acompañadas de un ejemplo de un reactivo de cada subescala.

Para determinar los factores asociados con la sintomatología depresiva se orientó la investigación a identificar las variables que predicen la sintomatología depresiva de los adolescentes michoacanos. Mediante el análisis de regresión múltiple con el método de pasos sucesivos se encontró una explicación del $43 \%$ de la varianza con la participación de siete variables; la de mayor peso fue la de dificultades en el manejo de la tristeza, seguida por el autocontrol, expresión en la familia, dificultades en el manejo del enojo, las dificultades para buscar apoyo, el sexo del estudiante y el tener madre migrante. En estos resultados se encuentran tanto recursos personales como familiares y sociales, además de variables que contemplan la migración familiar (cuadro 2).

Como puede apreciarse en el cuadro 3, y siendo consistentes con el constructo teórico del instrumento, las escalas de sintomatología depresiva presentan correlaciones estadísticamente significativas entre sí, con dirección positiva y valores medios y altos. Respecto a los recursos psicológicos, en el caso del autocontrol éste se correlaciona significativamente de manera negativa y baja con todas las subescalas de la CESD-R. Por el contrario, las subescalas de dificultades para el manejo de la tristeza y el enojo se correlacionan de manera positiva con las subescalas del CESD-R. Las dimensiones de dificultades en la familia, red de apoyo y recuperación del equilibrio no presentaron correlaciones significativas con las subescalas de sintomatología depresiva.

De los 360 participantes, 164 (45.6\%) tenían algún familiar migrante directo, ya fuera el padre, la madre o un hermano o hermana; la migración de familiares no directos o provenientes de su familia extensa como primos, tíos, sobrinos, cuñados, la presentaron 142 participantes (39.4\%). Sólo 54 (15\%) estudiantes reportaron no tener familiares migrantes. En la figura 1 puede apreciarse que en las cinco escalas de sintomatología depresiva evaluadas, quienes tienen familiares migrantes directos presentan mayores puntajes de sintomatología depresiva que quienes no tienen la experiencia de migración en la familia. Esas diferencias son estadísticamente significativas en el caso de la disforia $(\bar{X}=0.72$ y $\bar{X}=1.05, t=-2.736, \mathrm{gl}=114.5, \mathrm{p}=.01)$, el aislamiento $(\bar{X}=0.69$ y $\bar{X}=0.96, t=-2.18, g l=105.8, p=.041)$ y la ideación suicida $(\bar{X}=0.41$ y $\bar{X}=0.70, t=-2.065$, gl=116.451, $\mathrm{p}=.040)$.

Existen diferencias estadísticamente significativas por sexo en cuatro de las cinco subescalas de sintomatología depresiva. En todos los casos las mujeres presentan mayores puntajes de sintomatología depresiva que los varones (cuadro 4). De igual manera las mujeres presentan mayores dificultades en el manejo del enojo y de la tristeza. En contraste, en ellas se encuentran puntajes significativamente mayores de red de apoyo que en los varones. En las subescalas de anhedonia y en los recursos afectivos de autocontrol, manejo de la tristeza, en los recursos sociales de dificultades para pedir 

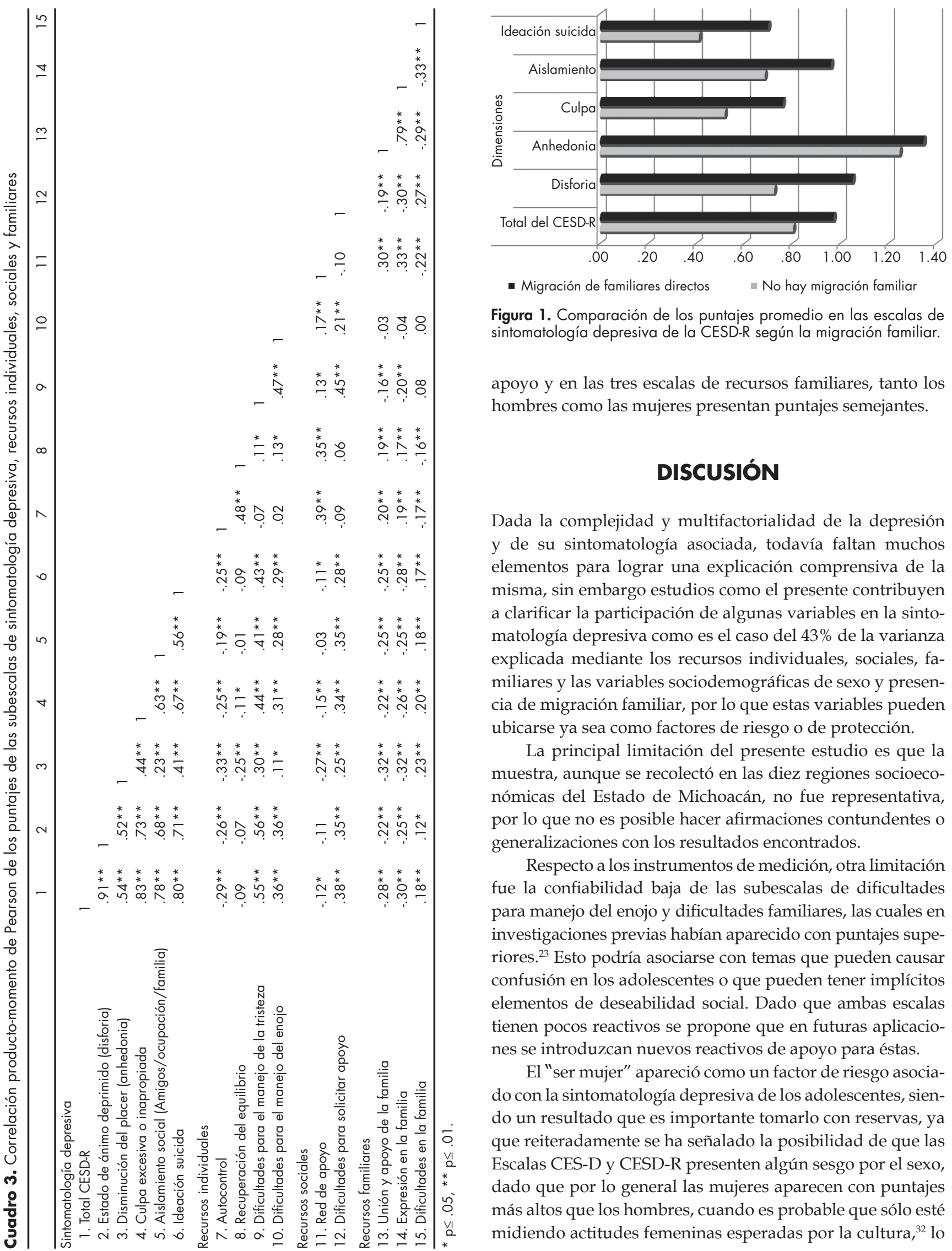

Figura 1. Comparación de los puntajes promedio en las escalas de sintomatología depresiva de la CESD-R según la migración familiar.

apoyo y en las tres escalas de recursos familiares, tanto los hombres como las mujeres presentan puntajes semejantes.

\section{DISCUSIÓN}

Dada la complejidad y multifactorialidad de la depresión y de su sintomatología asociada, todavía faltan muchos elementos para lograr una explicación comprensiva de la misma, sin embargo estudios como el presente contribuyen a clarificar la participación de algunas variables en la sintomatología depresiva como es el caso del $43 \%$ de la varianza explicada mediante los recursos individuales, sociales, familiares y las variables sociodemográficas de sexo y presencia de migración familiar, por lo que estas variables pueden ubicarse ya sea como factores de riesgo o de protección.

La principal limitación del presente estudio es que la muestra, aunque se recolectó en las diez regiones socioeconómicas del Estado de Michoacán, no fue representativa, por lo que no es posible hacer afirmaciones contundentes o generalizaciones con los resultados encontrados.

Respecto a los instrumentos de medición, otra limitación fue la confiabilidad baja de las subescalas de dificultades para manejo del enojo y dificultades familiares, las cuales en investigaciones previas habían aparecido con puntajes superiores. ${ }^{23}$ Esto podría asociarse con temas que pueden causar confusión en los adolescentes o que pueden tener implícitos elementos de deseabilidad social. Dado que ambas escalas tienen pocos reactivos se propone que en futuras aplicaciones se introduzcan nuevos reactivos de apoyo para éstas.

$\mathrm{El}$ "ser mujer" apareció como un factor de riesgo asociado con la sintomatología depresiva de los adolescentes, siendo un resultado que es importante tomarlo con reservas, ya que reiteradamente se ha señalado la posibilidad de que las Escalas CES-D y CESD-R presenten algún sesgo por el sexo, dado que por lo general las mujeres aparecen con puntajes más altos que los hombres, cuando es probable que sólo esté midiendo actitudes femeninas esperadas por la cultura, ${ }^{32}$ lo 
Rivera-Heredia et al.

Cuadro 4. Dimensiones de sintomatología depresiva y recursos individuales y sociales en donde se encontraron diferencias significativas por sexo

\begin{tabular}{lllllll}
\hline & Sexo & Media & DE & $\dagger$ & gl & Signif. \\
\hline Sintomatología depresiva & & & & & & \\
$\quad$ Disforia & mujeres & 1.23 & 0.95 & 6.62 & 358 & .001 \\
Culpa & varones & 0.68 & 0.59 & & & \\
& mujeres & 0.84 & 0.99 & 3.76 & 358 & .001 \\
Aislamiento & varones & 0.5 & 0.68 & & & \\
& mujeres & 1.03 & 0.92 & 3.79 & 358 & .001 \\
Ideación suicida & varones & 0.7 & 0.74 & & & \\
& mujeres & 0.9 & 1.23 & 5.92 & 358 & .001 \\
Recursos individuales (afectivos) & varones & 0.3 & 0.56 & & & \\
$\quad$ Dificultades para el manejo de la tristeza & mujeres & 2.45 & 0.76 & 7.01 & 356 & .001 \\
& varones & 1.92 & 0.66 & & & \\
Dificultades para el manejo del enojo & mujeres & 2.52 & 0.51 & 3.77 & 356 & .001 \\
& varones & 2.32 & 0.49 & & & \\
Recursos sociales & & & & & & \\
$\quad$ Red de apoyo & mujeres & 3.1 & 0.81 & 4.09 & 357 & \\
& varones & 2.75 & 0.82 & & & \\
\hline
\end{tabular}

cual ocurre con otros instrumentos diagnósticos sobre este mismo constructo, hecho que lleva a reflexionar tanto respecto a la nosología de la depresión como a la forma en que se ha estado midiendo.

Otro factor de riesgo observado en esta muestra de adolescentes fue "tener una madre migrante", lo cual arroja datos interesantes que dan elementos de apoyo para centrar la mirada en los familiares que se quedan y no en los migrantes en sí mismos. Por ejemplo, en estudios previos se había encontrado que existe más depresión en personas migrantes que en personas no migrantes; ${ }^{33}$ sin embargo todavía falta mayor investigación que permita conocer qué pasa con quienes se quedan a vivir y trabajar en su país pero tienen familiares migrantes. Por otro lado, dado que por muchos años la tradición migratoria mexicana fue tradicionalmente de varones, podría darse un efecto de "adaptación" a los "usos y costumbres" de la familia o región ante la migración del padre; sin embargo no ocurre lo mismo cuando la madre migra, según los resultados del presente trabajo en el que se encontró que la migración de la madre está estrechamente ligada a la sintomatología depresiva de los estudiantes participantes.

Hasta que no se cuente con mayor evidencia empírica, las explicaciones sobre la depresión en los migrantes que proporciona Bhugra ${ }^{26}$ podrían retomarse como hipótesis de trabajo que se apliquen en los adolescentes de la muestra estudiada que cuentan con una madre migrante, como el sentimiento de pérdida, el fatalismo, la vivencia de sucesos de vida negativos, menor desarrollo de las habilidades sociales, las contradicciones en los sistemas de creencias y de valores, entre otros, que podrían estar viviendo los adolescentes que se han quedado en sus comunidades de origen al estar expuestos a las influencias que tiene la migración de una persona tan central en la dinámica familiar mexicana, como ha sido tradicionalmente la madre, lo cual es un tema que se recomienda retomar en estudios posteriores.

Por otra parte, en la bibliografía científica se ha encontrado que a mayor estrés y menor apoyo social, se presenta mayor depresión. ${ }^{25}$ En el presente estudio se destaca que, más que carecer de una red de apoyo lo que ocurre con los adolescentes con sintomatología depresiva es que presentan dificultades para solicitar dicho apoyo a su red, lo cual coincide con lo encontrado en investigaciones previas..$^{22}$ Dichas dificultades pudieran relacionarse con la "emocionalidad restrictiva" (dificultad y miedo para expresar los propios sentimientos y emociones) que existe en los adolescentes que presentan síntomas de depresión e intento suicida, ${ }^{14}$ y que se observa en los adolescentes del presente estudio, quienes además reportan dificultades con el manejo del enojo y la tristeza.

Se encontró que un factor protector ante la sintomatología depresiva fue la "expresión en la familia", que implica la posibilidad de comunicar los sentimientos y emociones en un contexto de aceptación y respeto por parte de los integrantes de la misma. Este resultado es la contraparte de algunos factores familiares que se han identificado previamente como factores de riesgo ante la depresión tales como las dinámicas familiares conflictivas, caracterizadas por una comunicación agresiva o de rechazo, conflictos en los padres por el dinero, la falta de control parental o el exceso de éste y la escasa cohesión familiar. ${ }^{6,18-20}$

Se apuntala la necesidad de tener una visión ecológica para poner mayor atención a los ambientes donde los individuos se desenvuelven que permita comprender de mejor manera la sintomatología depresiva, los sucesos estresantes, así como los recursos con los que la enfrentan y salen ade- 
lante. Tal como lo propone Hobfoll ${ }^{24}$ es posible promover el desarrollo de los "recursos en caravanas" para lo que es necesario identificar cómo obtienen, conservan, incrementan, protegen o pierden los recursos que poseen, en la medida en que se van ajustando a sus diferentes contextos. Por ello proponemos que se diseñen investigaciones que analicen lo que ocurre en los adolescentes dentro de los contextos reales en los que se desenvuelven, incorporando estrategias de observación directa. Adicionalmente, resulta relevante incluir las diferencias culturales y poder trabajar de manera especializada por región, ya que así se obtendrá una intervención diseñada para las necesidades propias de cada localidad; intervenciones que partan desde la identificación, conservación, promoción y obtención de recursos psicológicos.

Los resultados de la presente investigación pueden retomarse para el desarrollo de propuestas de intervención específicas para la población con mayor riesgo (por ejemplo, programas para mujeres, o para jóvenes con migración familiar directa, especialmente de la madre), además de apuntalar factores protectores que se recomienda fortalecer en los adolescentes. Como recomendación central se encuentra el trabajar con los recursos afectivos de los adolescentes, desarrollando en ellos una mayor habilidad para el manejo de la tristeza y del enojo así como un mayor autocontrol. En el caso de los recursos sociales se requiere apoyarles en el desarrollo de habilidades para solicitar ayuda a su red de apoyo y en cuanto a los recursos familiares, se recomienda promover la expresión de emociones y sentimientos en la familia desde una postura de aceptación y respeto de sus integrantes. Corresponde a los profesionales de la salud, de las ciencias sociales, educadores, padres de familia e investigadores, continuar con este trabajo.

\section{AGRADECIMIENTOS}

Agradecemos el apoyo brindado por la Secretaría de Educación Pública a través del programa de mejoramiento al profesorado al proyecto de investigación "Promoción de la salud y prevención de conductas problema en estudiantes de secundaria" PROMEPUMSNH-252 así como a la Secretaría de Educación Pública del Estado de Michoacán que facilitó el acceso a las escuelas secundarias. También se agradece a los egresados de la Facultad de Psicología de la Universidad Michoacana de San Nicolás de Hidalgo que colaboraron en este proyecto: Diana Paulina Escutia, Héctor Hurtado, Patricia Rodríguez, Ángeles Durán, Criceida Pedro, Olivia Zamora, Laura Hernández Alegre, Monserrat García, Zeila Chávez, Brenda Corona, Ligia Velázquez, Lizbeth Sánchez, Margarita Vega, Miriam Roxana Mora y Maricarmen Gómez.

\section{REFERENCIAS}

1. World Health Organization (WHO). Mental Health. Depression. Recuperado el 29 de agosto de 2011 de http://www.who.int/mental_health/management/depression/definition/en/

2. Pardo G, Sandoval A, Umbarila D. Adolescencia y depresión. Rev Col Psicol 2004;13:17-42.
3. Wagner FA, Gallo JJ, Delva J. Depression in late life: A hidden public health for Mexico? Salud Publica Mex 1999;41:189-202.

4. Benjet C, Borges G, Medina-Mora ME, Zambrano J et al. Youth mental health in a populous city of the developing world: Results from the Mexican Adolescent Mental Health Survey. J Child Psychol Psychiatry 2009;50(4):386-395.

5. Radloff LS. Scale: A self-report depression scale for research in the general population (CES-D). Appl Psychol Meas 1977;1:385-401.

6. González-Forteza C, Jiménez-Tapia JA, Ramos-Lira L, Wagner FA. Aplicación de la Escala de Depresión del Center of Epidemiological Studies en adolescentes de la Ciudad de México. Salud Publica Mex 2008;50(4):292-299.

7. Reyes M, Soto AL, Milla JG, García A Et al. Actualización de la Escala de Depresión del Centro de Estudios Epidemiológicos (CES-D) en Poblaciones Geriátricas Mexicanas. Un estudio piloto. Salud Mental 2003;26(1):59-68.

8. García-Peña C, Wagner FA, Sánchez-García $S$, Juárez-Cedillo $T$ et al. A study of depressive symptoms among older adults in Mexico City. JGIM 2008;23:1973-1980.

9. Van Dam NT, Earleywine M. Validation of the Center for Epidemiologic Studies Depression Scale-Revised (CESD-R): Pragmatic depression assessment in the general population. Psychiatry Res 2011;186(1):128-132.

10. Chabrol H, Rodgers R, Rousseau A. Relations between suicidal ideation and dimensions of depressive symptoms in high-school students. J Adolesc 2007;30:587-600.

11. Andrade $P$, Betancourt $D$. Factores individuales, familiares y sociales y conductas de riesgo en adolescentes. En: Andrade P, Betancourt D, Cañas JL (eds.). Investigaciones psicosociales en adolescentes. México: Colección Montebello, UNICACH y UNAM; 2008.

12. Harrington R. Consecuencias psicosociales de la depresión adolescente. Psiquiatría Salud Integral 2001;1(2):48-52.

13. Vallejo A, Osorno R, Mazadiego T. Sintomatología depresiva medida con las CES-D en adolescentes del norte de Veracruz. En: Andrade $P$, Betancourt D, Cañas JL (eds.). Investigaciones psicosociales en adolescentes. México: Colección Montebello, UNICACH y UNAM; 2008.

14. Jacobson CM, Marrocco F, Kleinman M, Gould MS. Restrictive emotionality, depressive symptoms, and suicidal thoughts and behaviors among high school students. J Youth Adolescence 2011;40:656-665.

15. Ruelas E, Lozano R. ¿Juventud saludable? Este país. Tendencias Opiniones 2009;217:55-59.

16. Bagur AR. La atención de los jóvenes mexicanos. ¿Éxito o fracaso? Este país. Tendencias Opiniones 2009;217:4-14.

17. Álvarez-Zúniga M, Ramírez-Jacobo B, Silva-Rodríguez A, Coffin-Cabrera $\mathrm{N}$ et al. La relación entre depresión y conflictos familiares en adolescentes. International J Child Psychol Psychiatry 2009;9(02):205-216.

18. Galicia-Moyeda IX, Sánchez-Velasco A, Robles-Ojeda FJ. Factores asociados a la depresión en adolescentes: Rendimiento escolar y dinámica familiar. Anales Psicología 25(2):227-240.

19. Estévez E, Musitu G, Herrero J The influence of violent behavior and victimization at school on psychological distress: the role of parents and teachers. Adolescence 2005;40:183-195.

20. Monge-Holguín JA, Cubillas-Rodríguez MJ, Román-Pérez R, AbrilValdez E. Intentos de suicidio en adolescentes de educación media y superior y su relación con la familia. Psicología Salud 2007;17:45-51.

21. Rivera-Heredia, ME. Psicología y comunicación visual. Estrategias para la prevención del suicidio en adolescentes. México: Trillas; 2010.

22. Rivera-Heredia ME, Andrade P. Recursos individuales y familiares que protegen al adolescente del intento suicida. Rev Intercont Psicol Educ 2006;8(2):23-40.

23. Alvaro C, Lyons RF, Warner G, Hobfoll SE et al. Conservation of resources theory and research use in health systems. Implementation Science 2010;5(79):1-20.

24. Hobfoll SE. Conservation of resource caravans and engaged settings. J Occup Organ Psychol 2011;84:116-122. 
25. Vranceanu AM, Hobfoll H, Johnson R. Child multi-type maltreatment and associated depression and PTSD symptoms: The role of social support and stress. Child Abuse Negl 2007;31:71-84.

26. Bhugra D. Migration and depression. Acta Psychiatr Scand 2003;108(Suppl. 418):67-72.

27. Aguilera-Guzmán RM, Carreño-García MS, Juárez-García F. Características psicométricas de la CES-D en una muestra de adolescentes rurales mexicanos en zonas con alta tradición migratoria. Salud Publica Mex 2004;27(6):57-66.

28. González-Forteza C, Ramos-Lira L, Caballero-Gutiérrez MA, Wagner FA. Correlatos psicosociales de depresión, ideación e intento suicida en adolescentes mexicanos. Psicothema 2003; 15(4):524-532.

29. Mariño C, Medina-Mora ME, Chaparro A, González-Forteza C. Con- fiabilidad Y estructura factorial del CES-D en la muestra de adolescentes mexicanos. Rev Mex Psicol 1993;2:141-145.

30. Roberts E. Reliability of the CES-D: Scale in different ethnic contexts. Psychiatry Res 1980;2:125-134.

31. SPSS para Windows Versión 17.0. Illinois: SPSS INC. 2007.

32. Osorno-Munguía JR, Vallejo-Casarín A, Segura Celis-Ochoa B, Mazadiego-Infante TJ. Evaluación del funcionamiento diferencial de los ítems (DIF) de la Escala de Depresión del Centro para Estudios Epidemiológicos (CES-D) respecto del género en una muestra de adolescentes mexicanos. Rev Electr Psicol Iztacala 2008;11(2):138-152.

33. Borges G, Breslau J, Maxwell Su, Miller M et al. Immigration and Suicidal Behavior Among Mexicans and Mexican Americans. Am J Public Health 2009;99(4):728-733.

Artículo sin conflicto de intereses 